\title{
Modification and characterization of an aptamer-based surface plasmon resonance sensor chip
}

\author{
Junpeng $\operatorname{Tan}^{1,2,3}$, Bin Hao ${ }^{1,2}$, Zhicheng Liu ${ }^{1,2}$, Fengbo Bai ${ }^{4}$, Ruiqin Yang ${ }^{3, \mathrm{a}}$ and Hongxia Hao ${ }^{1,2, \mathrm{~b}}$ \\ ${ }^{1}$ Collaborative Innovation Center of Judicial Civilization, China \\ ${ }^{2}$ Key Laboratory of Evidence Science, China University of Political Science and Law, Ministry of Education, Beijing \\ 100088, China \\ ${ }^{3}$ People's Public Security University of China, Beijing 100038, China \\ ${ }^{4}$ Hong TeCH(Beijing)Co., Ltd, Beijing, 100021,China
}

\begin{abstract}
Recently, aptamer-based surface plasmon resonance (SPR) sensors have become increasingly popular due to their high specificity, high sensitivity, real-time detection capabilities, and label-free features. The core component of an aptamer-based SPR sensor is a chip. This paper presents the modification steps and the characterization results of a sensor chip for the construction of a 2, 4, 6-trinitrotoluene-targeted, aptamer-based, SPR sensor. After cleaning the aptamer-based SPR sensor chip, polyethylene glycol (PEG) with functional thiol groups at one end was added to the chip surface by Au-S covalent bonds to form a selfassembled film. Then, the carboxyl groups at the other end of PEG and the carboxyl groups of trinitrophenyl-glycine (TNP-Gly) were activated and connected via ethylenediamine (EDA). This effectively completed the chip's modification. During the modification process, relevant experimental conditions were optimized. The chip's surface elements, as well as their chemical states, were characterized by X-ray photoelectron spectroscopy (XPS). The results, outlined in the following study, demonstrate that this modification of an aptamer-based SPR sensor chip adhered to normative expectations. Thus, the modification process proposed here establishes an important foundation for subsequent study of TNT detection.
\end{abstract}

\section{Introduction}

As early as 1990, Tuerk and Ellington [1] independently screened RNA oligonucleotide aptamers out from oligonucleotide libraries. Aptamers can be defined as randomly obtained, single-stranded

\footnotetext{
${ }^{\text {a }}$ Corresponding author: Rqyang1971@yahoo.com

${ }^{\mathrm{b}}$ Corresponding author: haohx@126.com
}

We are grateful to Humanities and social science research program of China university of politics and law (10ZFQ82009) and Academician Foundation of the Ministry of Public Security of the People's Republic of China (No. 2011-23214203, 2011-23215243), Beijing Municipal Education Commission University Science and Technology Park Construction Project (2011-08111603) and Program for Young Innovative Research Team in China University of Political Science and Law (14CXTD04, 16CXTD05) for their financial supports. 
oligonucleotides that can bind specifically to target proteins and other small molecules through the systematic evolution of ligands by exponential enrichment (SELEX) technique [2, 3]. Aptamers can be classified as DNA, RNA, or even XNA, and their molecular weights are relatively small compared to common antibodies [4]. Aptamers have been applied and developed in biochemistry, sensor development, protein research, drug detection, and numerous other areas for many years [5-10].

Similar to the operation of antibodies, the screening targets of aptamers include all kinds of areas, from inorganic ions, amino acids, and organic dyes to proteins, drugs, and explosives. Based on SELEX technology, we can screen aptamers for any target molecules [11]. The affinity between an aptamer and its target molecule is exquisitely high, with a usual dissociation constant somewhere between $10^{-12} \mathrm{~mol} / \mathrm{L}$ and $10^{-9} \mathrm{~mol} / \mathrm{L}$ [12], much higher than common ligands. Moreover, aptamers are capable of distinguishing subtle differences between different ligands, and can even distinguish a single protein molecule's different configurations and/or functional states. Compared to the synthesis of antibodies, the preparation of aptamers only relies on in vitro screening of SELEX technology. Not only is this more cost-effective, but it also has other advantages, such as smaller molecule weights than antibodies. For this reason, it can be used for specific recognition of small molecules. In addition, aptamers show high stability during repeated screening, and they can be recycled in various external environments.

Due to their high affinity with target molecules, as well as their strong specificity, aptamers are potentially highly usable in fields such as analytical chemistry, gene detection, and drug development. However, since they cannot generate detection signals when bound to target substances, aptamers usually need the help of surface plasmon resonance (SPR), fluorescence detection [13], electrochemical detection [14], Raman spectroscopy [15], or colorimetry [16] to carry out signal conversion and detection. Among these technologies, a combination of an SPR sensor with aptamers for the detection of targets has recently become a research priority.

SPR is a physical and optical phenomenon. By measuring a metal surface's refractive index fluctuations, SPR can be used to study the optical properties of different materials [17]. Figure 1 shows the SPR sensor's basic principles [18]. When a polarized light beam hits the surface of a prism in a specific range of angles, an interface plasmon wave is generated between the prism's surface and the metal film (gold or silver). When the propagation constant of the incident light wave matches the propagation constant of the interface plasmon wave, the metal film's free electrons resonate (in other words, the surface plasmon resonates). During our analysis, we fixed a layer of a self-assembled film to the sensor chip's surface; the sample then flowed over the chip's surface. If there are molecules in the sample that can potentially bind to the self-assembled film, the refractive index of the gold film surface changes, leading to additional changes in the SPR resonance angle. The detection of these changes provides particular information, such as the analytes' concentration, affinity, kinetic constants, and specificity [19-23].

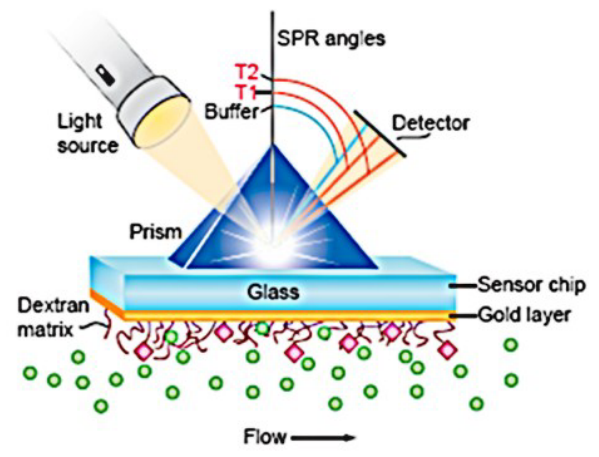

Figure 1. Schematic view for the principle of SPR sensor.

Based on the methodology of detecting 2, 4, 6-trinitrotoluene (TNT) with an aptamer-based, SPR sensor chip, our study focuses on the sensor's core, which was the modification and characterization 
of a trinitrophenyl-glycine (TNP-Gly) chip. In our process, we took advantage of the high specificity of aptamers and the high sensitivity of SPR, constructing an SPR sensor that could detect TNT directly and rapidly. Due to the stability of Au-S bonds [24, 25], TNP-Gly with carboxyl groups effectively and stably bound to the gold film surface with the help of modified polyethylene glycol (mPEG-SH) and the activation of 1-(3-dimethylaminopropyl)-3-ethylcarbodiimide hydrochloride/ Nhydroxylsuccinimide (EDC/NHS). Since polyethylene glycol (PEG) contains bifunctional groups (HS-PEG7-OH and HS-PEG7-COOH), the functional -SH groups were coupled to the SPR chip surface; NHS/EDC was used to activate the $-\mathrm{COOH}$ at the other end, and via amide reactions with ethylenediamine (EDA), the TNP-Gly with carboxyl groups was connected to the chip's surface. In this way, we were able to complete TNP-Gly chip synthesis. For the characterization process, an Xray photoelectron spectroscopy (XPS) was used to perform quantification analysis during each stage. Figure 2 presents the basic principles and synthesis methods.

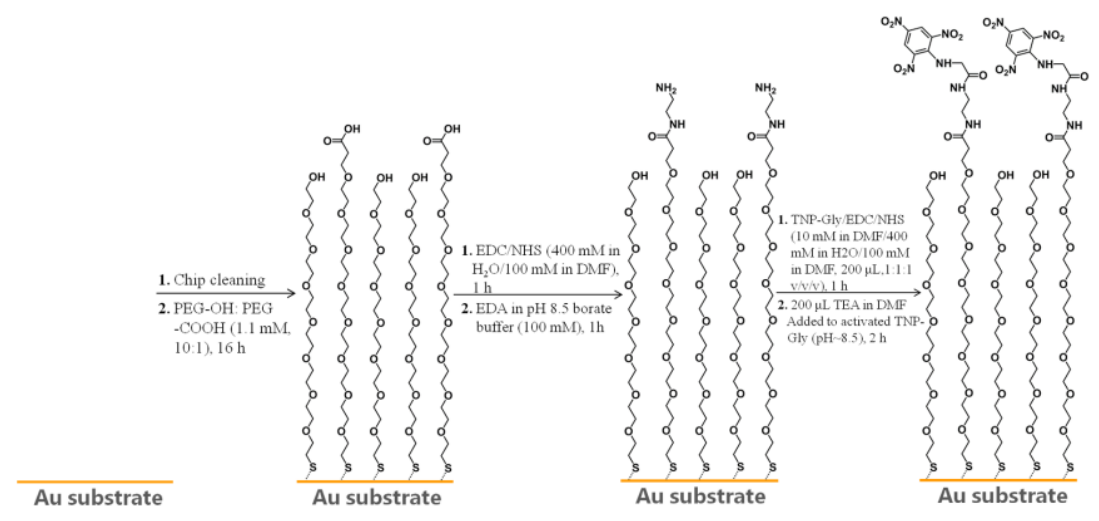

Figure 2. The basic principles and synthesis methods of the SPR sensor chip.

\section{Experiment}

\subsection{Materials and instruments}

The TNP-Gly we used was obtained from Research Plus (US). Thio PEG Hydroxyl (HS-PEG $-\mathrm{OH}$ ) and thio $\mathrm{PEG}$ carboxylic acid $\left(\mathrm{HS}-\mathrm{PEG}_{7}-\mathrm{COOH}\right)$ were obtained from Ploypure (Norway). Nhydroxysuccinimide (NHS) and 1-(3-dimethylaminopropyl)-3-ethylcarbodiimide hydrochloride $(\mathrm{EDC} \cdot \mathrm{HCl})$ were provided by from Sinopharm Chemical Reagent Co., Ltd. Sodium dodecyl sulfate (SDS) and triethylamine (TEA) were obtained from Shanghai Anpel Laboratory Technologies Inc. Ethylenediamine (EDA) and N, N-dimethylformamide (DMF) were obtained from Tianjin Fuchen Chemical Reagents Factory. Methanol, ethanol, and acetone were provided by Beijing Chemical Reagent Company.

Our X-ray photoelectron spectroscopy analyzer (XPS, ESCALAB 250Xi) was produced by Thermo Fisher Scientific Inc. The plasma cleaner (PDC-32G-2) was obtained from Mycro Technologies Co., Ltd. The shaker (cyclotron oscillator HY-5A) was from Ronghua Instrument Manufacturing Co., Ltd. The vortex (2E-161240) was manufactured by Scientific Industries Inc., US. The base for our aptamer-based, SPR sensor chip was made of BK7 optical glass $(2.5 \mathrm{~cm} \times 2.0 \mathrm{~cm} \times$ $0.1 \mathrm{~cm}$ ), with one side coated with $2 \mathrm{~nm}$ of chromium film and (afterwards) with $45 \mathrm{~nm}$ of gold film using a high vacuum coating method. 


\subsection{Chip cleaning}

Our method for the preparation of $1 \%$ SDS washing solution was as follows. a sample of $10 \mathrm{~g}$ SDS was added to a $100 \mathrm{~mL}$ beaker and diluted to $1 \mathrm{~L}$ in a volumetric flask. Cleaning the chip with $1 \%$ SDS solution: the gold-coated chip (by vacuum deposition) was placed in a glass dish, with the gold film side facing up. Then an appropriate amount of $1 \%$ SDS solution was added to soak and wash the chip three times by hand-shaking. The chip was then soaked in the SDS solution and shaken in a shaker at $45 \mathrm{r} / \mathrm{min}$ for $20 \mathrm{~min}$. Afterwards the chip was removed with a tweezer and rinsed off any residual SDS solution with deionized water, and dried with nitrogen. The chip was then placed in a clean glass dish with the gold film facing up, soaked with acetone, and washed by hand-shaking for another three times. Soaked in acetone, the chip was shaken at $45 \mathrm{r} / \mathrm{min}$ for $3 \mathrm{~min}$. Afterwards acetone was removed and ethanol was added to soak and wash the chip by hand-shaking for an additional three times. Soaked in ethanol, the chip was shaken at $45 \mathrm{r} / \mathrm{min}$ for $3 \mathrm{~min}$. Then ethanol was removed and methanol was added to soak and wash the chip by hand-shaking for another three times. Soaked in methanol, the chip was shaken at $45 \mathrm{r} / \mathrm{min}$ for $3 \mathrm{~min}$. After cleaning, the chip was removed with a tweezer and dried with nitrogen. The dried chip was cleaned further in a plasma cleaner at mediumlow level for $60 \mathrm{~s}$. Finally it was sealed and stored.

\subsection{Modification of the chip}

A sample of $10 \mu \mathrm{L}$ HS-PEG-OH and $10 \mu \mathrm{L}$ HS-PEG-COOH were respectively added to a $2 \mathrm{~mL}$ microtube. A volume of $1 \mathrm{~mL}$ deionized water was added into each tube and properly shaken, thus the stock solutions were prepared. A volume of $3.529 \mathrm{~mL}$ ethanol was added to a glass dish; $421 \mu \mathrm{L}$ HSPEG-OH stock solution and $50 \mu \mathrm{L}$ HS-PEG-COOH stock solution were added subsequently to make a molar ratio of 10:1 for PEG-OH: PEG-COOH. The stored chip was soaked into the above solution with the gold film facing up. After sealed, it was shaken at $45 \mathrm{r} / \mathrm{min}$ for $16 \mathrm{~h}$ to allow complete contact and reaction between the chip and PEG.

During the reaction, a sample of $0.6901 \mathrm{~g}$ EDC was dissolved in $3 \mathrm{~mL}$ deionized water and 0.1036 g NHS was dissolved in $3 \mathrm{~mL}$ DMF, to make a molar ratio of EDC: NHS $=4: 1$ later on. After $16 \mathrm{~h}$, the SPR chip in the glass dish was removed and first rinsed with ethanol, then cleaned with an ultrasonic cleaner for $5 \mathrm{~s}$, and finally dried with nitrogen. In a clean glass dish, $1.333 \mathrm{~mL}$ deionized water, $1.333 \mathrm{~mL}$ EDC stock solution and $1.333 \mathrm{~mL}$ NHS stock solution were added, then the dried chip was soaked in and shaken at $45 \mathrm{r} / \mathrm{min}$ for $1 \mathrm{~h}$.

During the EDC/NHS activation period, EDA stock solution was prepared: in $3.737 \mathrm{~mL}$ borate buffer ( $\mathrm{pH}$ 8.5), 13-14 drops of EDA reagent were added dropwise. After the activation reaction, the SPR chip was removed, rinsed with deionized water, and dried with nitrogen. In a new glass dish, a $500 \mu \mathrm{L}$ EDA stock solution and $3.5 \mathrm{~mL}$ borate buffer $(\mathrm{pH} \mathrm{8.5)}$ were combined, then the above dried chip was soaked in this solution and shaken at $45 \mathrm{r} / \mathrm{min}$ for $1 \mathrm{~h}$. Afterwards, the SPR chip was once again removed, rinsed with deionized water, and dried with nitrogen.

During the reaction between the chip and the EDA solution, a TNP-Gly stock solution was prepared: a sample of $0.0056 \mathrm{~g}$ TNP-Gly was dissolved in $400 \mu \mathrm{L}$ DMF. Then $200 \mu \mathrm{L}$ of the above TNP-Gly solution was transferred to a clean glass dish, and combined with $200 \mu \mathrm{L}$ EDC stock solution, $100 \mu \mathrm{L}$ NHS stock solution, and $100 \mu \mathrm{L}$ DMF successively. After mixed uniformly, it was shaken at $45 \mathrm{r} / \mathrm{min}$ for $1 \mathrm{~h}$. The TEA solution was prepared as well: 16 drops of TEA were added to $400 \mu \mathrm{L}$ DMF. After the TNP-Gly solution was shaken for $1 \mathrm{~h}$, it was added with $200 \mu \mathrm{L}$ TEA solution and mixed well to prepare the TNP-Gly stock solution. The $600 \mu \mathrm{L}$ TNP-Gly stock solution was then transferred to cover the gold film surface of the prepared chip with a $1 \mathrm{~mL}$ transfer pipette. After kept still for $2 \mathrm{~h}$, a plastic head dropper was used to remove all the TNP-Gly stock solution along the chip's edge. The chip was rinsed with deionized water, dried with nitrogen, and stored. 


\section{Results and discussion}

\subsection{Chosen of chip modifiers}

PEG is soluble in water, methanol, and benzene, while it is insoluble in ether and n-hexane. Due to its special structure, both ends of PEG can be modified by a variety of functional group types to meet different needs. Additionally, as a polymer, PEG's relative molecular weight varies depending on its polymerization degree. A variety of PEGs with different molecular weights have been widely applied to excipients in the pharmaceutical industry, stabilizers in the chemical industry, matrix in the textile and cosmetics industry, among others. In laboratory studies on the surface modification of gold nanoparticles, PEG is normally used to stabilize the electrons in the nanoparticle surface.

After an extensive review of the relevant literature, our lab decided to use the mPEG-SH type of PEG as our gold film surface modifier [26-29]. The primary reasons for this decision were as follows. (1) The gold film deposited by our group was a naked gold film with only three elements on its surface: C, O, and Au. In order to make the chip's surface TNP-Gly modifiable, it need to be modified and activated first. It was then more conducive to further modification with compounds such as EDC/NHS and EDA. (2) PEG is antigen-free, immunogenic-free, non-toxic, and biocompatible, qualities that are particularly important for subsequent binding with aptamers. (3) In physiological environments, PEG has no adsorption of or interactions with other biological molecules. Therefore, we estimated that it would not cause interference of elements from external environments when coupled to the gold film surface. This was crucial for the XPS analysis, since it ensured the high stability of gold film. (4) Based on PEG'S advantage of having two ends that can be linked to functional groups, our study selected mPEG-SH-type PEG (HS-PEG $\mathrm{P}_{7}-\mathrm{OH}$ and $\mathrm{HS}-\mathrm{PEG}_{7}-\mathrm{COOH}$ ) with strong activity and with average molecular weights within the PEG400 category. Due to Au's high affinity with $\mathrm{S}$ on one end, it is coupled to the chip surface stably, leaving the other end with a functional group for stabilizing further modification. After testing $\mathrm{HS}_{-} \mathrm{PEG}_{7}-\mathrm{OH}$ : $\mathrm{HS}-\mathrm{PEG}_{7}-\mathrm{COOH}$ with ratios of $5: 2,4: 1,6: 1,10: 1$, and $20: 1$, as Figure 3 shows, the 10:1 molar ratio was chosen to modify the gold film chip..

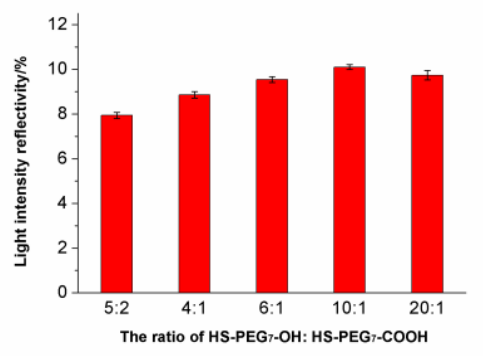

Figure 3. The SPR response signal of five ratios of $\mathrm{HS}-\mathrm{PEG}_{7}-\mathrm{OH}: \mathrm{HS}-\mathrm{PEG}_{7}-\mathrm{COOH}$.

\subsection{Chosen of chip activator}

$\mathrm{EDC} \cdot \mathrm{HCl}$ is soluble in water and often serves as a dehydrating agent to react with a carboxyl group. NHS is soluble in water and DMF, and it is usually used as a condensing agent to improve condensation efficiency in EDC reactions. These two elements are often used together to activate carboxyl groups to form acyl groups, and then to replace the hydrogens of amino groups to produce amides in amide reactions.

During the modification process of the chip's gold film, PEG with -COOH was used. Therefore, as Figure 2 shows, there were - $\mathrm{COOH}$ groups in the chip's surface after modification. The TNP-Gly to be modified, on the other hand, carried amino groups. Therefore, EDC/NHS was chosen to serve as 
the activator for the coupling reaction [30-33]. As shown in Figure 4, consulting the test results of different EDC/NHS ratios, the ratio of EDC: $\mathrm{NHS}=4: 1$ was chosen for further experiments.

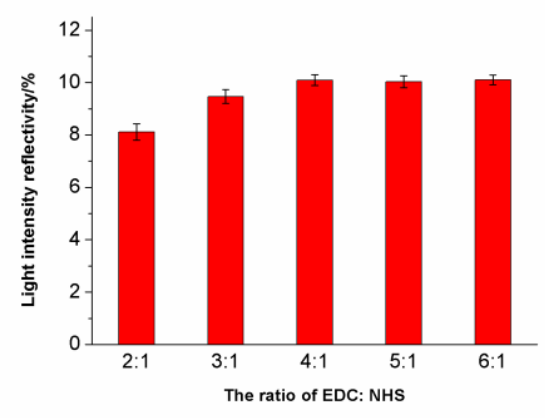

Figure 4. The SPR response signal of different ratios of EDC: NHS.

\subsection{Chosen of reaction time and $\mathrm{pH}$}

Since the whole synthesis process was broken down into four stages, the chosen of reaction time and $\mathrm{pH}$ for each stage was very important. The reaction did not require a special temperature, therefore, room temperature was used throughout the whole experiment.

Stage I, as Figure 2 shows below, consisted of coating the chip with HS-PEG-OH and HS-PEG$\mathrm{COOH}$. Since the gold film had a strong $\mathrm{S}$ affinity, the formation of $\mathrm{Au}-\mathrm{S}$ bonds did not require special external conditions except for a thorough reaction time. This study used a ratio of HS-PEG$\mathrm{OH}$ : HS-PEG-COOH $=10: 1$ to bind to the naked chip. Due to the large area of the gold film $(20 \mathrm{~mm}$ $\times 15 \mathrm{~mm} \times 1 \mathrm{~mm}$ ), there were less $-\mathrm{SH}$ groups from PEG than Au elements on the chip's surface.

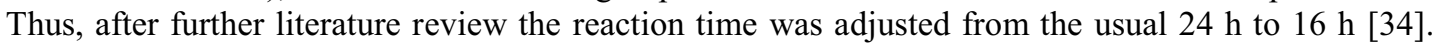
Moreover, to avoid high $\mathrm{pH}$ interference on the activation reaction during the introduction of EDC/NHS later on, the reaction between PEG and the chip was in an ethanol solution rather than the alkaline SDS solution usually used for PEG modification. In this experiment, SDS was only used during cleaning and deionization of the naked chip.

Stage II consisted of EDC/NHS activation and EDA introduction. In carboxyl-modified experiments, EDC usually achieves the best result between $\mathrm{pH} 4$ and $\mathrm{pH} 6$, due to its own structural characteristics. However, for the modified $\mathrm{HS}_{-} \mathrm{PEG}_{7}-\mathrm{OH}$ and $\mathrm{HS}-\mathrm{PEG}_{7}-\mathrm{COOH}$, a weak alkaline environment was required to keep PEG activity. Later, during the TNP-Gly linking process, a weak alkaline environment was also required to avoid the non-coupling of TNP-Gly. Therefore, the carboxyl activation for the gold film was performed under a neutral $\mathrm{pH}$, with a reaction time of $1 \mathrm{~h}$ (slightly longer than usual). To avoid any external interference during TNP-Gly coupling, EDA was introduced. During the PEG modification and EDC/NHS activation process, the acyl groups were significantly excessive compared to the resulting TNP-Gly. Therefore, to avoid the interference of external compounds and groups due to excessive acryl, EDA with double amino groups were used to neutralize the acryl groups. To keep the reaction in a weak alkaline environment, ${ }_{\mathrm{p}} \mathrm{H} 8.5$ borate buffer was used, with a reaction time of $1 \mathrm{~h}$.

Stages III and IV included the preparation of the TNP-Gly stock solution and its binding to the modified chip. To ensure complete binding between TNP-Gly and the chip, EDC/NHS was added into the TNP-Gly stock solution. Following both the literature references and our own tests, the reaction time for the binding of TNP-Gly to the chip was set to $2 \mathrm{~h}$. 


\subsection{Characterization and analysis of the sensor chip}

During the synthesis of the chip and TNP-Gly, the unmodified chip, the PEG-modified chip, the EDC/NHS-activated chip, and the fully modified chip were all analyzed with XPS to record the binding of the modified process at each stage. Results are shown below.

\subsubsection{Unmodified chip}

The XPS analysis of the unmodified chip demonstrated that only C, O, and Au elements were detected on the chip's surface (see Table 1). This indicated that, after cleaning with SDS, organic solvents, and plasma cleaner, any interferences from $\mathrm{N}, \mathrm{S}$, and other elements were successfully removed. The chip was therefore completely clean before modification. Figure 5 shows the XPS spectra of the three elements mentioned above.

Table 1. Element contents of the unmodified chip.

\begin{tabular}{|c|c|c|}
\hline Element & Peak Value & Content (\%) \\
\hline $\mathrm{C}_{1 \mathrm{~s}}$ & 285.03 & 30.27 \\
\hline $\mathrm{O}_{1 \mathrm{~s}}$ & 532.33 & 14.40 \\
\hline $\mathrm{Au}_{4 \mathrm{f}}$ & 84.07 & 53.64 \\
\hline
\end{tabular}
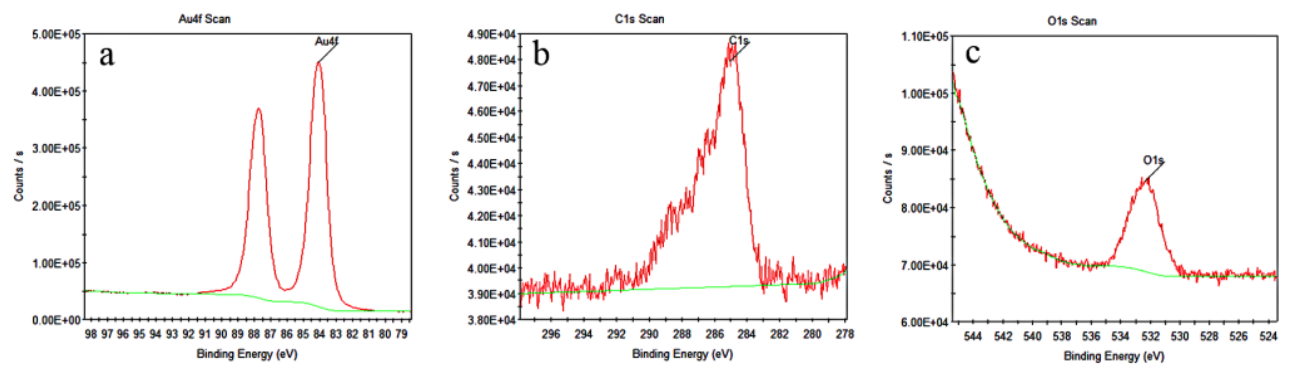

Figure 5. The XPS spectra of three elements of the unmodified chip (a:Au-SCAN, b: C-SCAN, c: O-SCAN).

\subsubsection{PEG-modified chip}

Table 2 shows the element contents from the XPS analysis of the PEG-modified chip. These results demonstrated that $\mathrm{S}$ appeared on the chip's surface, indicating the coupling of S elements to the gold film. Since the PEG we used contained -SH groups, PEG had formed stable Au-S bonds on the gold film surface (see Figure 6a). In Figure $6 \mathrm{~b}$ it is clear that the element content of $\mathrm{C}$ was elevated compared to the unmodified chip, and that there was an $\mathrm{O}-\mathrm{C}=\mathrm{O}$ absorption peak at $288.5 \mathrm{eV}$ and a $\mathrm{C}$ $\mathrm{O}-\mathrm{C}$ absorption peak at $286.7 \mathrm{eV}$. As Figure $6 \mathrm{c}$ shows, the element content of $\mathrm{O}$ was also increased compared to the unmodified chip, and there was a $\mathrm{C}=\mathrm{O}$ absorption peak at $533 \mathrm{eV}$. Our results suggested the existence of $-\mathrm{COOH}$ groups on the gold film surface. Therefore, it can be concluded that HS- $\mathrm{PEG}_{7}-\mathrm{COOH}$ and $\mathrm{HS}-\mathrm{PEG}_{7}-\mathrm{OH}$ were coupled to the chip's surface through Au-S bonds, and the surface of the gold film was successfully modified with $\mathrm{PEG}$ with - $\mathrm{OH}$ and $-\mathrm{COOH}$ groups, forming an effective $\mathrm{Au}-\mathrm{OEG}_{7} \mathrm{OH}: \mathrm{OEG}_{7} \mathrm{COOH}$ structure. 
Table 2. Element contents of the PEG-modified chip.

\begin{tabular}{|c|c|c|}
\hline Element & Peak Value & Content (\%) \\
\hline $\mathrm{C}_{1 \mathrm{~s}}$ & 286.70 & 42.96 \\
\hline $\mathrm{O}_{1 \mathrm{~s}}$ & 532.91 & 23.11 \\
\hline $\mathrm{Au}_{4 \mathrm{f}}$ & 84.01 & 31.05 \\
\hline $\mathrm{S}_{2 \mathrm{p}}$ & 162.23 & 2.44 \\
\hline $\mathrm{Zn}_{2 \mathrm{p} 3}$ & 1022.34 & 0.45 \\
\hline
\end{tabular}
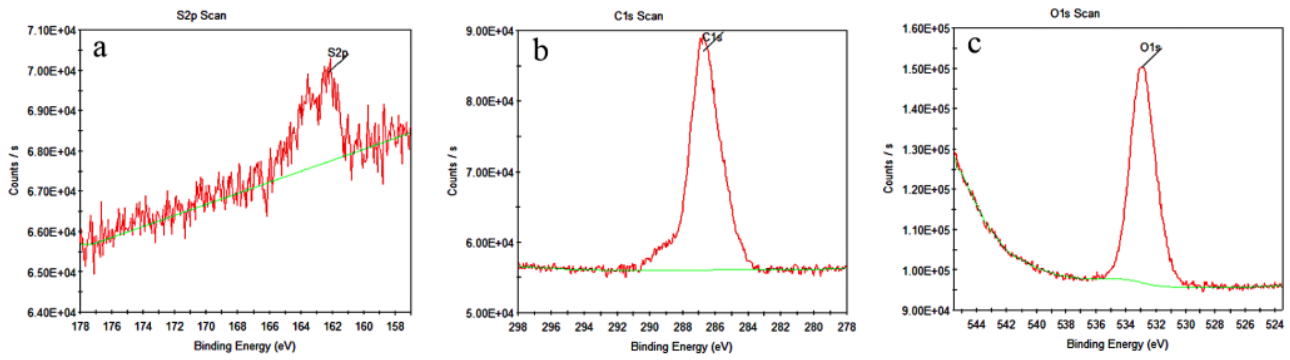

Figure 6. The XPS spectra of three elements of the PEG-modified chip (a:S-SCAN, b: C-SCAN, c: O-SCAN).

\subsubsection{EDC/NHS-activated chip}

The XPS analysis has been performed for the EDC/NHS-activated chip. Table 3 shows the element contents, while Figure 7 presents the XPS spectra of the S, N, C, and O. A new element N appeared during detection on the chip's surface, indicating successful coupling of $\mathrm{N}$ elements to the chip's surface. Moreover, there was a $\mathrm{C}-\mathrm{NH} 2$ absorption peak at $400 \mathrm{eV}$ but no $\mathrm{O}-\mathrm{C}=\mathrm{O}$ absorption peak at $288.5 \mathrm{eV}$, suggesting that the original $-\mathrm{COOH}$ on the chip underwent amino reactions with EDA after EDC/NHS activation. Thus, the original $-\mathrm{COOH}$ disappeared and only $\mathrm{C}-\mathrm{NH} 2$ groups could be found on the chip's surface, forming an Au-OEG7CO-NH $(\mathrm{CH} 2 \mathrm{CH} 2) \mathrm{NH} 2$ structure. 
Table 3. Element contents of the EDC/NHS-activated chip.

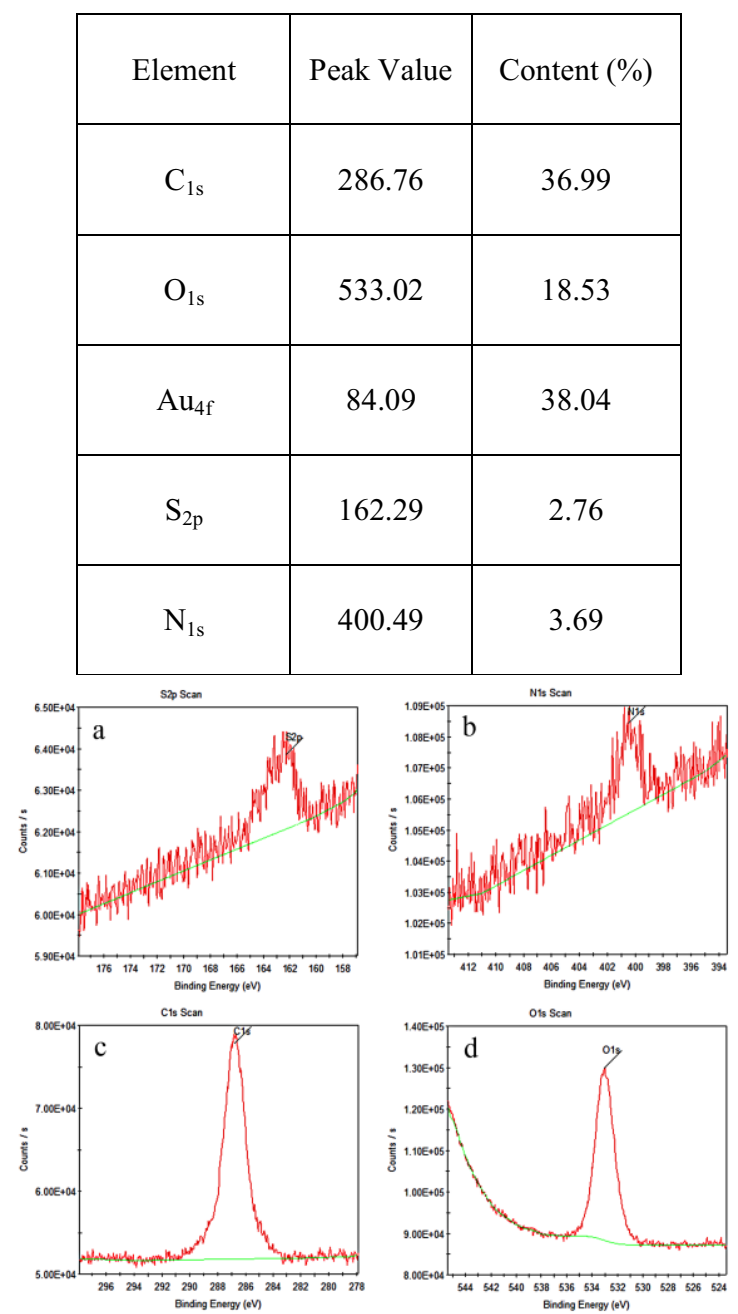

Figure 7. The XPS spectra of four elements of the EDC/NHS-activated chip (a:S-SCAN, b: N-SCAN, c: CSCAN, d:O- SCAN).

\subsubsection{Fully modified chip}

The XPS analysis has been performed for the fully modified chip. Table 4 shows the element contents, which exhibited no significant change from the EDC/NHS-activated chip. Figure 8 presents the XPS spectra of the $\mathrm{S}, \mathrm{N}, \mathrm{C}$, and $\mathrm{O}$. There was a $-\mathrm{NO}_{2}$ absorption peak at $406 \mathrm{eV}$ and a $\mathrm{C}-\mathrm{NH}_{2}$ peak at 400 $\mathrm{eV}$. The $-\mathrm{NO}_{2}$ came from TNP-Gly and the C-NH2 came from both TNP-Gly and EDA. Our results indicated that $\mathrm{Au}-\mathrm{OEG}_{7} \mathrm{CO}-\mathrm{NH}\left(\mathrm{CH}_{2} \mathrm{CH}_{2}\right) \mathrm{N}-\mathrm{Gly}-\mathrm{TNP}$ formed on the chip's surface. 
Table 4. Element contents of the fully modified chip.

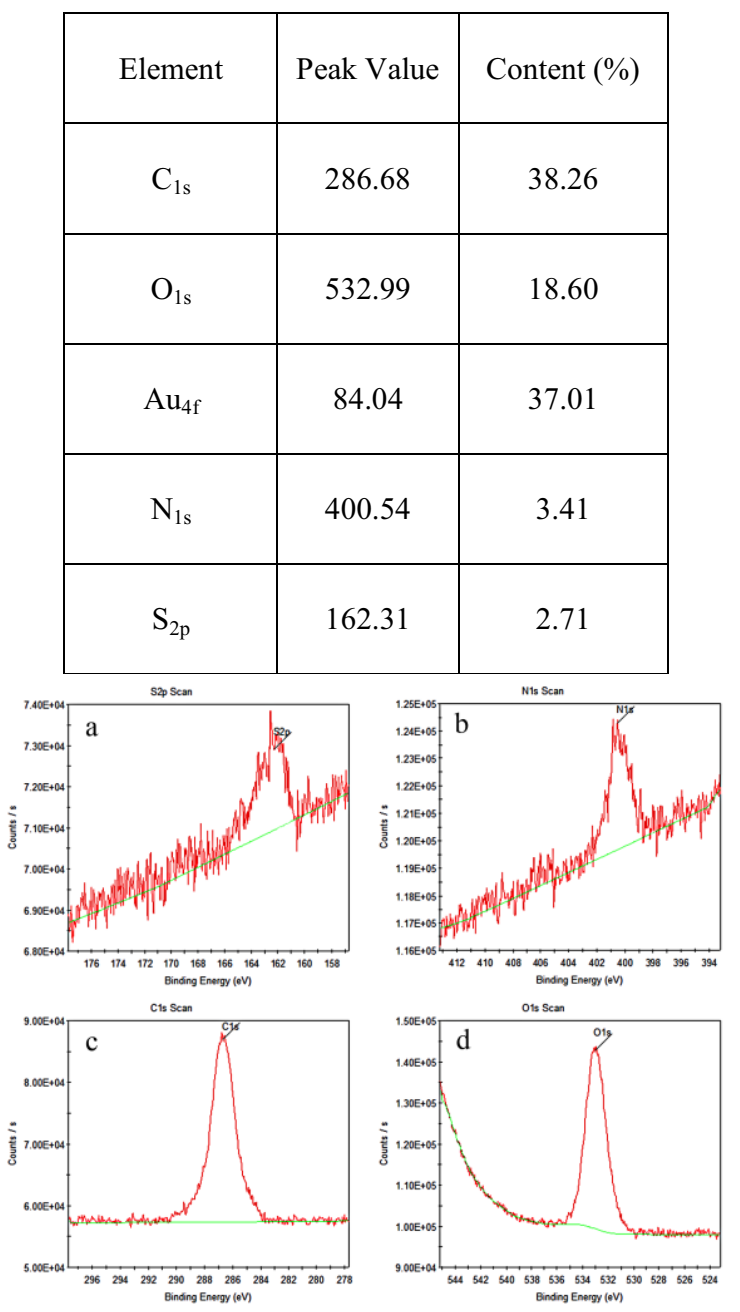

Figure 8. The XPS spectra of four elements of the fully modified chip (a:S-SCAN, b: N-SCAN, c: C-SCAN, d:O- SCAN).

\section{Conclusion}

Taking advantage of the stable binding between -SH and Au, as well as the PEG quality of having two ends that can bind to functional groups, this study used the mPEG-SH type of PEG that contains both $-\mathrm{COOH}$ and $-\mathrm{OH}$ groups to bind to a chip. Following EDC/NHS activation, this type of PEG connected to the amino-containing TNP-Gly to assemble the TNP-Gly chip. During our experimental design, the main difficulty we encountered was in selecting the appropriate ratio between HS-PEG$\mathrm{OH}$ and HS-PEG-COOH. To solve this difficulty, the ratios of 4:1, 5:2, 7:3, 10:1, and 20:1 were tested with the XPS spectra at different ratios for each stage. Based on our own results and a comprehensive literature review, the ratio of 10:1 was chosen for further modification, due to its superior performance. After coupling with TNP-Gly, the XPS spectra showed an expected $-\mathrm{NO}_{2}$ absorbance peak. The successful chip synthesis we present in this study establishes a solid foundation for further investigation of TNT detection. 


\section{References}

1. A.D. Ellington, J.W. Szostak. Nature, 346(6872), 8818-8822 (1990).

2. S Song, L Wang, J Li, et al. Trac-Trend Anal Chen, 27(2), 108-117(2008).

3. W Sun, L.M. Du. Curr. Pharm. Design, 16(20), 2269-2278(2010).

4. C. Tuerk, L. Gold. Science, 249(4968), 505-510 (1990).

5. H.R. Jang, A.W. Wark, S.H. Baek, et al. Anal Chen, 86(1), 814-819(2013).

6. P. Subramanian, A. Lesniewski, I. Kaminska, et al. Biosens Bioelectron, 50C(24), 239-243(2013).

7. H. Bai, R. Wang, B. Hargis, et al. Sensors, 12(9), 12506-12518(2011).

8. M.Y. Kim, S. Jeong. Nucleic Acid Therapeutics, 21(3), 173-178(2011).

9. P. Janardhanan, C.M. Mello, B.R. Singh, et al. Talanta, 117(24), 273-280(2013).

10. B. Prieto-Simón, J. Samitier. Anal Chen, 86(3), 1437-1444(2014).

11. S. Tombelli, M. Minunni, M. Mascini, Biosens Bioelectron, 20(12), 2424-2434(2005).

12. N. Chelyapov. Biochemistry, 45(7), 2461-2466 (2006).

13. R. Nutiu, Y.F. Li. J Am Chen Soc, 125(16), 4771-4778 (2003).

14. XL Zuo, SP Song, J Zhang, et al. JACS, 129(5), 1042-1043 (2007).

15. J.W. Chen, J.H. Jiang, X. Gao, et al. Chem. Eur. J, 14(27), 8374-8382(2008).

16. R. Jin, G. Wu, Z. Li, et al. JACS, 125(6), 1643-1654(2003).

17. R.W. Wood. Proceedings of the Physical Society of London, 18(1), 269-275(1902).

18. P. Englebienne, A.V. Hoonacker, M Verhas. Spectrosc-Int J, 17(2-3), 255-273(2003).

19. S. Sonezaki, S Yagi, E Ogawa, et al. J Immunol Methods, 238(238), 99-106(2000).

20. W. Lee, D.B. Lee, B.K. Oh, et al. Enzyme Microb Tech, 35(6-7), 678-682(2004).

21. J. Homola, J. Dostálek, S. Chen, et al. Int J Food Microbiol, 75(1-2), 61-69(2002).

22. J. Wei, Y. Mu, D. Song, et al. Anal Biochem, 321(2), 209-216(2003).

23. D.R. Shankaran, K.V. Gobi, T. Sakai, et al. Biosens Bioelectron, 20(9), 1750-1756(2005).

24. T.M.H. And, M.J. Tarlov. J Am Chen Soc, 119(38), 8916-8920(1997).

25. J. Wang, R. Lv, J. Xu, et al. Anal and Bioanal Chem, 390(4), 1059-1065(2008).

26. M. Yasuura, K. Toko, T. Onodera. Sensor Mater, 23(1), 21-37(2011).

27. T. Onodera, Y. Mizuta, K. Horikawa, et al. Sensor Mater, 23(1), 39-52(2011).

28. K. Nagatomo, T. Kawaguchi, N. Miura, et al. Talanta, 79, 1142-1148(2009).

29. T. Kawaguchi, D.R. Shankaran, S.J. Kim, et al. Talanta, 72, 554-560(2007).

30. I. Coille, G. Gauglitz, J. Hoebeke. Anal Bioanal Chem, 372, 293-300(2002).

31. I. Rault, V. Frei, D. Herbage, et al. J Mater Sci Mater Med, 7(7), 215-221(1996).

32. J.M. Lee, H.H.L. Edwards, C.A. Pereira, et al. J Mater Sci Mater Med, 7(9), 531-541(1996).

33. L.H.H.O. Damink, P.J. Dijkstra, M.J.A.V. Luyn, et al. Biomaterials, 17(7), 679-684(1996).

34. J. He, F. Zhao, C. Wu, et al. J Mater Chem B, 1(40), 5398-5402(2013). 